Coupled fixed point theorems and applications to periodic boundary value problems

\author{
Cristina Urs
}




\title{
COUPLED FIXED POINT THEOREMS AND APPLICATIONS TO PERIODIC BOUNDARY VALUE PROBLEMS
}

\author{
CRISTINA URS
}

Received 18 September, 2012

\begin{abstract}
In this paper we present some existence, uniqueness and Hyers-Ulam stability results for the coupled fixed point of a pair of contractive type operators on complete metric spaces. The approach is based on a Perov type fixed point theorem for contractions. Some applications to integral equations and to boundary value problems are also given.
\end{abstract}

2000 Mathematics Subject Classification: 47H10; 54H25

Keywords: coupled fixed point, singlevalued operator, vector-valued metric, periodic boundary value problem.

\section{INTRODUCTION}

The classical Banach contraction principle is a very useful tool in nonlinear analysis with many applications to operatorial equations, fractal theory, optimization theory and other topics. Banach contraction principle was extended for singlevalued contraction on spaces endowed with vector-valued metrics by Perov in [7], while the case of multivalued contractions is treated in A. Petruşel [8].

In the study of the existence of fixed points for an operator, it is useful to consider a more general concept, namely coupled fixed points. The concept of coupled fixed point for continuous and discontinuous operators was introduced in 1987 by D. Guo and V. Lakshmikantham (see [5]) in connection with coupled quasisolutions of an initial value problem for ordinary differential equations.

Let $X$ be a nonempty set. A mapping $d: X \times X \rightarrow \mathbb{R}^{m}$ is called a vector-valued metric on $X$ if the following properties are satisfied:

(a) $d(x, y) \geq 0$ for all $x, y \in X$; if $d(x, y)=0$, then $x=y$;

(b) $d(x, y)=d(y, x)$ for all $x, y \in X$;

(c) $d(x, y) \leq d(x, z)+d(z, y)$ for all $x, y \in X$.

This work was made possible with the financial support of the Sectoral Operational Programme for Human Resources Development 2007 - 2013, co-financed by the European Social Fund, under the project number POSDRU/107/1.5/S/76841 with the title Modern Doctoral Studies: Internationalization and Interdisciplinarity. 
A set endowed with a vector-valued metric $d$ is called generalized metric space. The notions of convergent sequence, Cauchy sequence, completeness, open subset and closed subset are similar to those for usual metric spaces.

We denote by $M_{m m}\left(\mathbb{R}_{+}\right)$the set of all $m \times m$ matrices with positive elements and by $I$ the identity $m \times m$ matrix. If $x, y \in \mathbb{R}^{m}, x=\left(x_{1}, \ldots, x_{m}\right)$ and $y=\left(y_{1}, \ldots, y_{m}\right)$, then, by definition:

$$
x \leq y \text { if and only if } x_{i} \leq y_{i} \text { for } i \in\{1,2, \ldots, m\} .
$$

Notice that we will make an identification between row and column vectors in $\mathbb{R}^{m}$.

For the proof of the main results we need the following theorems. A classical result in matrix analysis is the following theorem (see [1], [10], [13]).

Theorem 1. Let $A \in M_{m m}\left(\mathbb{R}_{+}\right)$. The following assertions are equivalents:

(i) $A$ is convergent towards zero;

(ii) $A^{n} \rightarrow 0$ as $n \rightarrow \infty$;

(iii) The eigenvalues of $A$ are in the open unit disc, i.e $|\lambda|<1$, for every

$\lambda \in \mathbb{C}$ with $\operatorname{det}(A-\lambda I)=0$;

(iv) The matrix $(I-A)$ is nonsingular and

$$
(I-A)^{-1}=I+A+\ldots+A^{n}+\ldots ;
$$

(v) The matrix $(I-A)$ is nonsingular and $(I-A)^{-1}$ has nonnegative elements;

(vi) $A^{n} q \rightarrow 0$ and $q A^{n} \rightarrow 0$ as $n \rightarrow \infty$, for each $q \in \mathbb{R}^{m}$.

We recall now Perov's fixed point theorem (see [7]).

Theorem 2 (Perov). Let $(X, d)$ be a complete generalized metric space and the operator $f: X \rightarrow X$ with the property that there exists a matrix $A \in M_{m m}(\mathbb{R})$ such that $d(f(x), f(y)) \leq A d(x, y)$ for all $x, y \in X$.

If $A$ is a matrix convergent towards zero, then:

(i) $\operatorname{Fix}(f)=\left\{x^{*}\right\}$;

(ii) the sequence of successive approximations $\left(x_{n}\right)_{n \in \mathbb{N}}, x_{n}=f^{n}\left(x_{0}\right)$ is convergent and has the limit $x^{*}$, for all $x_{0} \in X$;

(i i i) one has the following estimation

$$
d\left(x_{n}, x^{*}\right) \leq A^{n}(I-A)^{-1} d\left(x_{0}, x_{1}\right) ;
$$

(iv) if $g: X \rightarrow X$ is an operator such that there exist $y^{*} \in F i x(g)$ and $\eta \in$ $\left(\mathbb{R}_{+}^{m}\right)^{*}$ with $d(f(x), g(x)) \leq \eta$, for each $x \in X$, then

$$
d\left(x^{*}, y^{*}\right) \leq(I-A)^{-1} \eta ;
$$

(v) if $g: X \rightarrow X$ is an operator and there exists $\eta \in\left(\mathbb{R}_{+}^{m}\right)^{*}$ such that 
$d(f(x), g(x)) \leq \eta$, for all $x \in X$, then for the sequence $y_{n}:=g^{n}\left(x_{0}\right)$ we have the following estimation

$$
d\left(y_{n}, x^{*}\right) \leq(I-A)^{-1} \eta+A^{n}(I-A)^{-1} d\left(x_{o}, x_{1}\right) .
$$

For related results to Perov's fixed point theorem and for some generalizations and applications of it we refer to [3], [4], [9].

Let $(X, d)$ be a metric space. We will focus our attention to the following system of operatorial equations:

$$
\left\{\begin{array}{l}
u=A(u, v) \\
v=A(v, u)
\end{array}\right.
$$

where $A: X \times X \rightarrow X$ is a given operator.

By definition, a solution $(u, v) \in X \times X$ of the above system is called a coupled fixed point for $A$ (see also [5], [6]).

In this paper we present some coupled fixed points results for contractive type singlevalued operators on spaces endowed with vector-valued metrics and, as an application, we discuss the existence, uniqueness and Hyers-Ulam stability of the solution of a periodic boundary value problem related to a system of differential equations. The approach is based on Perov-type fixed point theorem for contractions in metric spaces endowed with vector-valued metrics.

\section{EXISTENCE, UNIQUENESS AND STABILITY RESULTS FOR COUPLED FIXED POINTS}

Definition 1. Let $(X, d)$ be a generalized metric space and $f: X \rightarrow X$ be an operator. Then, the fixed point equation

$$
x=f(x)
$$

is said to be generalized Hyers-Ulam stable if there exists an increasing function $\psi: \mathbb{R}_{+}^{m} \rightarrow \mathbb{R}_{+}^{m}$, continuous in 0 with $\psi(0)=0$, such that, for any $\varepsilon:=\left(\varepsilon_{1}, \ldots, \varepsilon_{m}\right)$ with $\varepsilon_{i}>0$ for $i \in\{1, \ldots, m\}$ and any solution $y^{*} \in X$ of the inequation

$$
d(y, f(y)) \leq \varepsilon
$$

there exists a solution $x^{*}$ of (2.1) such that

$$
d\left(x^{*}, y^{*}\right) \leq \psi(\varepsilon) .
$$

In particular, if $\psi(t)=C \cdot t, t \in \mathbb{R}_{+}^{m}$ (where $C \in M_{m m}\left(\mathbb{R}_{+}\right)$), then the fixed point equation (2.1) is called Hyers-Ulam stable.

We remind a direct consequence of Perov's fixed point theorem.

Theorem 3. Let $(X, d)$ be a generalized metric space and let $f: X \rightarrow X$ be an operator with the property that there exists a matrix $A \in M_{m m}(\mathbb{R})$ such that $A$ converges to zero and

$$
d(f(x), f(y)) \leq A d(x, y), \text { for all } x, y \in X .
$$


Then the fixed point equation

$$
x=f(x), x \in X
$$

is Hyers-Ulam stable.

Definition 2. Let $(X, d)$ be a metric space and let $T_{1}, T_{2}: X \times X \rightarrow X$ be two operators. Then the operatorial equations system

$$
\left\{\begin{array}{l}
x=T_{1}(x, y) \\
y=T_{2}(x, y)
\end{array}\right.
$$

is said to be Hyers-Ulam stable if there exist $c_{1}, c_{2}, c_{3}, c_{4}>0$ such that for each $\varepsilon_{1}, \varepsilon_{2}>0$ and each solution-pair $\left(u^{*}, v^{*}\right) \in X \times X$ of the inequations:

$$
\begin{aligned}
& d\left(u^{*}, T_{1}\left(u^{*}, v^{*}\right)\right) \leq \varepsilon_{1} \\
& d\left(v^{*}, T_{2}\left(u^{*}, v^{*}\right)\right) \leq \varepsilon_{2}
\end{aligned}
$$

there exists a solution $\left(x^{*}, y^{*}\right) \in X \times X$ of (2.4) such that

$$
\begin{aligned}
& d\left(u^{*}, x^{*}\right) \leq c_{1} \varepsilon_{1}+c_{2} \varepsilon_{2} \\
& d\left(v^{*}, y^{*}\right) \leq c_{3} \varepsilon_{1}+c_{4} \varepsilon_{2}
\end{aligned}
$$

For related results regarding Hyers-Ulam stability of the operatorial equations see [11].

We recall the following existence, uniqueness, data dependence and Hyers-Ulam stability theorem for the coupled fixed point of a pair of singlevalued operators (see [12] ).

Theorem 4. Let $(X, d)$ be a complete metric space, $T_{1}, T_{2}: X \times X \rightarrow X$ such that

$$
\begin{aligned}
& d\left(T_{1}(x, y), T_{1}(u, v)\right) \leq k_{1} d(x, u)+k_{2} d(y, v) \\
& d\left(T_{2}(x, y), T_{2}(u, v)\right) \leq k_{3} d(x, u)+k_{4} d(y, v)
\end{aligned}
$$

for all $(x, y),(u, v) \in X \times X$. We suppose that $A=\left(\begin{array}{ll}k_{1} & k_{2} \\ k_{3} & k_{4}\end{array}\right)$ converges to zero. Then

(i) there exists a unique element $\left(x^{*}, y^{*}\right) \in X \times X$ such that

$$
\left\{\begin{array}{l}
x^{*}=T_{1}\left(x^{*}, y^{*}\right) \\
y^{*}=T_{2}\left(x^{*}, y^{*}\right)
\end{array}\right.
$$

(ii) the sequence $\left(T_{1}^{n}(x, y), T_{2}^{n}(x, y)\right)_{n \in \mathbb{N}}$ converges to $\left(x^{*}, y^{*}\right)$ as $n \rightarrow \infty$, where

$$
\begin{aligned}
& T_{1}^{n+1}(x, y):=T_{1}^{n}\left(T_{1}(x, y), T_{2}(x, y)\right) \\
& T_{2}^{n+1}(x, y):=T_{2}^{n}\left(T_{1}(x, y), T_{2}(x, y)\right)
\end{aligned}
$$

for all $n \in \mathbb{N}^{*}$. 
(iii) we have the following estimation

$$
\left(\begin{array}{c}
d\left(T_{1}^{n}\left(x_{0}, y_{0}\right), x^{*}\right) \\
d\left(T_{2}^{n}\left(x_{0}, y_{0}\right), y^{*}\right)
\end{array}\right) \leq A^{n}(I-A)^{-1}\left(\begin{array}{c}
d\left(x_{0}, T_{1}\left(x_{0}, y_{0}\right)\right) \\
d\left(y_{0}, T_{2}\left(x_{0}, y_{0}\right)\right)
\end{array}\right)
$$

(iv) let $F_{1}, F_{2}: X \times X \rightarrow X$ be two operators such that, there exist $\eta_{1}, \eta_{2}>0$ with

$$
\begin{aligned}
& d\left(T_{1}(x, y), F_{1}(x, y)\right) \leq \eta_{1} \\
& d\left(T_{2}(x, y), F_{2}(x, y)\right) \leq \eta_{2}
\end{aligned}
$$

for all $(x, y) \in X \times X$. If $\left(a^{*}, b^{*}\right) \in X \times X$ is such that

$$
\left\{\begin{array}{l}
a^{*}=F_{1}\left(a^{*}, b^{*}\right) \\
b^{*}=F_{2}\left(a^{*}, b^{*}\right)
\end{array}\right.
$$

then

$$
\left(\begin{array}{l}
d\left(a^{*}, x^{*}\right) \\
d\left(b^{*}, y^{*}\right)
\end{array}\right) \leq(I-A)^{-1} \eta
$$

where $\eta:=\left(\begin{array}{l}\eta_{1} \\ \eta_{2}\end{array}\right)$

(v) let $F_{1}, F_{2}: X \times X \rightarrow X$ be two operators such that, there exist $\eta_{1}, \eta_{2}>0$ with

$$
\begin{aligned}
& d\left(T_{1}(x, y), F_{1}(x, y)\right) \leq \eta_{1} \\
& d\left(T_{2}(x, y), F_{2}(x, y)\right) \leq \eta_{2}
\end{aligned}
$$

for all $(x, y) \in X \times X$ and considering the sequence $\left(F_{1}^{n}(x, y), F_{2}^{n}(x, y)\right)_{n \in \mathbb{N}}$, where

$$
\begin{aligned}
& F_{1}^{n+1}(x, y):=F_{1}^{n}\left(F_{1}(x, y), F_{2}(x, y)\right) \\
& F_{2}^{n+1}(x, y):=F_{2}^{n}\left(F_{1}(x, y), F_{2}(x, y)\right)
\end{aligned}
$$

for all $n \in \mathbb{N}^{*}$ and $\eta:=\left(\begin{array}{l}\eta_{1} \\ \eta_{2}\end{array}\right)$ then

$$
\begin{gathered}
\left(\begin{array}{c}
d\left(F_{1}^{n}\left(x_{0}, y_{0}\right), x^{*}\right) \\
d\left(F_{2}^{n}\left(x_{0}, y_{0}\right), y^{*}\right)
\end{array}\right) \leq(I-A)^{-1} \eta+A^{n}(I-A)^{-1} . \\
\left(\begin{array}{c}
d\left(x_{0}, T_{1}\left(x_{0}, y_{0}\right)\right) \\
d\left(y_{0}, T_{2}\left(x_{0}, y_{0}\right)\right)
\end{array}\right)
\end{gathered}
$$

(vi) the operatorial equations system

$$
\left\{\begin{array}{l}
x=T_{1}(x, y) \\
y=T_{2}(x, y)
\end{array}\right.
$$

is Hyers-Ulam stable. 


\section{AN APPLICATION TO PERIODIC BOUNDARY VALUE PROBLEM}

In this section we study the existence, uniqueness and Hyers-Ulam stability of a solution to a periodic boundary value problem as an application of the coupled fixed point Theorem 4 presented in Section 2. The approach is based on the application presented in [2].

We consider the periodic boundary value problem

$$
\left\{\begin{array}{c}
u^{\prime}=f(t, u)+g(t, v) \\
v^{\prime}=f(t, v)+g(t, u) \\
u(0)=u(T) \\
v(0)=v(T)
\end{array}\right.
$$

assuming that $f, g$ are continuous functions and satisfy the following conditions:

Assumption 1. There exist $\lambda_{1}>0, \lambda_{2}>0$ and $\mu_{1}>0, \mu_{2}>0$, such that for all $u, v \in \mathbb{R}, v \leq u$,

$$
\begin{aligned}
0 & \leq\left(f(t, u)+\lambda_{1} u\right)-\left(f(t, v)+\lambda_{1} v\right) \leq \mu_{1}(u-v) \\
-\mu_{2}(u-v) & \leq\left(g(t, u)-\lambda_{2} u\right)-\left(g(t, v)-\lambda_{2} v\right) \leq 0,
\end{aligned}
$$

where $S:=\left(\begin{array}{cc}\frac{\mu_{1}}{\lambda_{1}+\lambda_{2}} & \frac{\mu_{2}}{\lambda_{1}+\lambda_{2}} \\ \frac{\mu_{2}}{\lambda_{1}+\lambda_{2}} & \frac{\mu_{1}}{\lambda_{1}+\lambda_{2}}\end{array}\right)$ is a matrix convergent to zero.

We study the existence of a solution of the following periodic system:

$$
\begin{aligned}
u^{\prime}+\lambda_{1} u-\lambda_{2} v & =f(t, u)+g(t, v)+\lambda_{1} u-\lambda_{2} v, \\
v^{\prime}+\lambda_{1} v-\lambda_{2} u & =f(t, v)+g(t, u)+\lambda_{1} v-\lambda_{2} u,
\end{aligned}
$$

together with the periodicity conditions,

$$
u(0)=u(T) \text { and } v(0)=v(T) .
$$

This problem is equivalent to the integral equations:

$$
\begin{aligned}
u(t)= & \int_{0}^{T} G_{1}(t, s)\left[f(s, u)+g(s, v)+\lambda_{1} u-\lambda_{2} v\right] \\
& +G_{2}(t, s)\left[f(s, v)+g(s, u)+\lambda_{1} v-\lambda_{2} u\right] d s \\
v(t)= & \int_{0}^{T} G_{1}(t, s)\left[f(s, v)+g(s, u)+\lambda_{1} v-\lambda_{2} u\right] \\
& +G_{2}(t, s)\left[f(s, u)+g(s, v)+\lambda_{1} u-\lambda_{2} v\right] d s
\end{aligned}
$$

where 


$$
\begin{gathered}
G_{1}(t, s)=\left\{\begin{array}{c}
\frac{1}{2}\left[\frac{e^{\sigma_{1}(t-s)}}{1-e^{\sigma_{1} T}}+\frac{e^{\sigma_{2}(t-s)}}{1-e^{\sigma_{2} T}}\right] 0 \leq s<t \leq T \\
\frac{1}{2}\left[\frac{e^{\sigma_{1}(t+T-s)}}{1-e^{\sigma_{1} T}}+\frac{e^{\sigma_{2}(t+T-s)}}{1-e^{\sigma_{2} T}}\right] 0 \leq t<s \leq T
\end{array}\right. \\
G_{2}(t, s)=\left\{\begin{array}{c}
\frac{1}{2}\left[\frac{e^{\sigma_{2}(t-s)}}{1-e^{\sigma_{2} T}}-\frac{e^{\sigma_{1}(t-s)}}{1-e^{\sigma_{1} T}}\right] 0 \leq s<t \leq T \\
\frac{1}{2}\left[\frac{e^{\sigma_{2}(t+T-s)}}{1-e^{\sigma_{2} T}}-\frac{e^{\sigma_{1}(t+T-s)}}{1-e^{\sigma_{1} T}}\right] 0 \leq t<s \leq T
\end{array}\right.
\end{gathered}
$$

Here, $\sigma_{1}=-\left(\lambda_{1}+\lambda_{2}\right)$ and $\sigma_{2}=\left(\lambda_{2}-\lambda_{1}\right)$.

We need to guarantee that $G_{1}(t, s) \geq 0,0 \leq t, s \leq T$, and $G_{2}(t, s) \leq 0,0 \leq t, s \leq T$, by choosing $\lambda_{1}, \lambda_{2}$ suitably. The following lemma addresses this issue.

Lemma 1 (Lakshmikantham, [2]). If

$$
\begin{aligned}
\ln \left(\frac{2 e-1}{e}\right) & \leq\left(\lambda_{2}-\lambda_{1}\right) T \\
\left(\lambda_{1}+\lambda_{2}\right) T & \leq 1
\end{aligned}
$$

then $G_{1}(t, s) \geq 0$ for $0 \leq t, s \leq T$, and $G_{2}(t, s) \leq 0$ for $0 \leq t, s \leq T$.

Let $X=C(I, \mathbb{R})$ be the metric space of all continuous functions $u: I \rightarrow \mathbb{R}$, endowed with the metric $d(u, v)=\sup _{t \in I}|u(t)-v(t)|$, for $u, v \in X$.

For $x, y, u, v \in X$, we also denote $\widetilde{d}((x, y),(u, v)):=\left(\begin{array}{c}d(x, u) \\ d(y, v)\end{array}\right)$

Let us define $A: X \times X \rightarrow X$ for $t \in I$, by

$$
\begin{aligned}
A(u, v)(t) & =\int_{0}^{T} G_{1}(t, s)\left[f(s, u)+g(s, v)+\lambda_{1} u-\lambda_{2} v\right] \\
& +G_{2}(t, s)\left[f(s, v)+g(s, u)+\lambda_{1} v-\lambda_{2} u\right] d s
\end{aligned}
$$

Note that if $(u, v) \in X \times X$ is a coupled fixed point of $A$, then we have

$$
u(t)=A(u, v)(t) \text { and } v(t)=A(v, u)(t), \text { for all } t \in I .
$$

Thus, $(u, v)$ is a solution of (3.4)-(3.6).

For the proof of our main result we need the following notion. 
Definition 3. The system

$$
\left\{\begin{array}{c}
u(t)=\int_{0}^{T} G_{1}(t, s)\left[f(s, u)+g(s, v)+\lambda_{1} u-\lambda_{2} v\right] \\
+G_{2}(t, s)\left[f(s, v)+g(s, u)+\lambda_{1} v-\lambda_{2} u\right] d s \\
v(t)=\int_{0}^{T} G_{1}(t, s)\left[f(s, v)+g(s, u)+\lambda_{1} v-\lambda_{2} u\right] \\
+G_{2}(t, s)\left[f(s, u)+g(s, v)+\lambda_{1} u-\lambda_{2} v\right] d s
\end{array}\right.
$$

is said to be Hyers-Ulam stable if there exist $c_{1}, c_{2}>0$ such that for each $\varepsilon_{1}, \varepsilon_{2}>0$ and each solution $\left(x^{*}, y^{*}\right)$ of the following inequation system

$$
\left\{\begin{array}{c}
\mid x^{*}(t)-\int_{0}^{T} G_{1}(t, s)\left[f\left(s, x^{*}\right)+g\left(s, y^{*}\right)+\lambda_{1} x^{*}-\lambda_{2} y^{*}\right] \\
+G_{2}(t, s)\left[f\left(s, y^{*}\right)+g\left(s, x^{*}\right)+\lambda_{1} y^{*}-\lambda_{2} x^{*}\right] d s \mid \leq \varepsilon_{1} \\
\mid y^{*}(t)-\int_{0}^{T} G_{1}(t, s)\left[f\left(s, y^{*}\right)+g\left(s, x^{*}\right)+\lambda_{1} y^{*}-\lambda_{2} x^{*}\right] \\
+G_{2}(t, s)\left[f\left(s, x^{*}\right)+g\left(s, y^{*}\right)+\lambda_{1} x^{*}-\lambda_{2} y^{*}\right] d s \mid \leq \varepsilon_{2}
\end{array}\right.
$$

there exists a solution $\left(u^{*}, v^{*}\right)$ of (3.9) such that

$$
\begin{aligned}
& \left|u^{*}(t)-x^{*}(t)\right| \leq c_{1} \varepsilon_{1}+c_{2} \varepsilon_{2} \\
& \left|v^{*}(t)-y^{*}(t)\right| \leq c_{3} \varepsilon_{1}+c_{4} \varepsilon_{2}
\end{aligned}
$$

Our main result is the following existence, uniqueness and Hyers-Ulam stability of a solution to a periodic boundary value problem.

Theorem 5. Consider the problem (3.1) with $f, g \in C(I \times \mathbb{R}, \mathbb{R})$ and suppose that the Assumption 1 is satisfied. If (3.7) and (3.8) are fulfilled, then:

(i) there exists a unique solution $\left(u^{*}, v^{*}\right)$ of the periodic boundary value problem (3.1).

(ii) let $f_{1}, g_{1} \in C(I \times \mathbb{R}, \mathbb{R})$ such that, there exist $\eta_{1}, \eta_{2}>0$ with

$$
\left\{\begin{array}{l}
\left|f(t, u)-f_{1}(t, u)\right| \leq \eta_{1} \\
\left|g(t, u)-g_{1}(t, u)\right| \leq \eta_{2}
\end{array}\right.
$$

for all $(t, u) \in I \times \mathbb{R}$. Let $\left(a^{*}, b^{*}\right) \in X \times X$ be a solution of the problem (3.1) with $f$ replaced by $f_{1}$ and $g$ replaced by $g_{1}$. Then

$$
\begin{gathered}
\tilde{d}\left(\left(u^{*}, v^{*}\right),\left(a^{*}, b^{*}\right)\right)=\left(\begin{array}{c}
d\left(a^{*}, u^{*}\right) \\
d\left(b^{*}, v^{*}\right)
\end{array}\right) \leq(I-S)^{-1} \eta \\
\text { where } \eta:=\left(\begin{array}{c}
\left(\eta_{1}+\eta_{2}\right) \frac{1}{\lambda_{2}-\lambda_{1}} \\
\left(\eta_{1}+\eta_{2}\right) \frac{1}{\lambda_{2}-\lambda_{1}}
\end{array}\right) .
\end{gathered}
$$

(iii) the system (3.9) is Hyers-Ulam stable. 
Proof. (i)

$$
\begin{aligned}
& d(A(x, y), A(u, v))=\sup _{t \in I}|A(x, y)(t)-A(u, v)(t)| \\
& =\sup _{t \in I} \int_{0}^{T} G_{1}(t, s)\left[f(s, x)+g(s, y)+\lambda_{1} x-\lambda_{2} y\right] \\
& +G_{2}(t, s)\left[f(s, y)+g(s, x)+\lambda_{1} y-\lambda_{2} x\right] d s \\
& -\int_{0}^{T} G_{1}(t, s)\left[f(s, u)+g(s, v)+\lambda_{1} u-\lambda_{2} v\right] \\
& +G_{2}(t, s)\left[f(s, v)+g(s, u)+\lambda_{1} v-\lambda_{2} u\right] d s \\
& =\sup _{t \in I} \int_{0}^{T} G_{1}(t, s) \\
& {\left[\left[f(s, x)+g(s, y)+\lambda_{1} x-\lambda_{2} y\right]-\left[f(s, u)+g(s, v)+\lambda_{1} u-\lambda_{2} v\right]\right]} \\
& +G_{2}(t, s)\left[\left[f(s, y)+g(s, x)+\lambda_{1} y-\lambda_{2} x\right]-\left[f(s, v)+g(s, u)+\lambda_{1} v-\lambda_{2} u\right]\right] d s \\
& =\sup _{t \in I} \int_{0}^{T} G_{1}(t, s) \\
& {\left[\left[f(s, x)+g(s, y)+\lambda_{1} x-\lambda_{2} y\right]-\left[f(s, u)+g(s, v)+\lambda_{1} u-\lambda_{2} v\right]\right]} \\
& -G_{2}(t, s)\left[\left[f(s, v)+g(s, u)+\lambda_{1} v-\lambda_{2} u\right]-\left[f(s, y)+g(s, x)+\lambda_{1} y-\lambda_{2} x\right] d s\right. \\
& \leq \sup _{t \in I} \int_{0}^{T} G_{1}(t, s)\left[\mu_{1}(x-u)+\mu_{2}(v-y)\right]-G_{2}(t, s)\left[\mu_{1}(v-y)+\mu_{2}(x-u)\right] d s \\
& =\left[\mu_{1} d(x, u)+\mu_{2} d(y, v)\right] \cdot \sup _{t \in I}\left|\int_{0}^{T}\left[G_{1}(t, s)-G_{2}(t, s)\right] d s\right| \\
& =\left[\mu_{1} d(x, u)+\mu_{2} d(y, v)\right] \cdot \sup _{t \in I}\left|\int_{0}^{t} \frac{e^{\sigma_{1}(t-s)}}{1-e^{\sigma_{1} T}} d s+\int_{t}^{T} \frac{e^{\sigma_{1}(t+T-s)}}{1-e^{\sigma_{1} T}} d s\right| \\
& =\frac{\mu_{1}}{\lambda_{1}+\lambda_{2}} d(x, u)+\frac{\mu_{2}}{\lambda_{1}+\lambda_{2}} d(y, v) \text {. }
\end{aligned}
$$

In a similar way we get that

$$
d(A(y, x), A(v, u)) \leq \frac{\mu_{1}}{\lambda_{1}+\lambda_{2}} d(y, v)+\frac{\mu_{2}}{\lambda_{1}+\lambda_{2}} d(x, u)
$$

If we denote $k_{1}:=\frac{\mu_{1}}{\lambda_{1}+\lambda_{2}}$ and $k_{2}:=\frac{\mu_{2}}{\lambda_{1}+\lambda_{2}}$, we get that

$$
\begin{aligned}
& d(A(x, y), A(u, v)) \leq k_{1} d(x, u)+k_{2} d(y, v) \quad \text { and } \\
& d(A(y, x), A(v, u)) \leq k_{2} d(x, u)+k_{1} d(y, v)
\end{aligned}
$$


Then we have

$$
\begin{aligned}
& \left(\begin{array}{c}
d(A(x, y), A(u, v)) \\
d(A(y, x), A(v, u))
\end{array}\right) \leq\left(\begin{array}{c}
k_{1} d(x, u)+k_{2} d(y, v) \\
k_{2} d(x, u)+k_{1} d(y, v)
\end{array}\right) \\
= & \left(\begin{array}{ll}
k_{1} & k_{2} \\
k_{2} & k_{1}
\end{array}\right)\left(\begin{array}{l}
d(x, u) \\
d(y, v)
\end{array}\right)=S \cdot \widetilde{d}((x, y),(u, v))
\end{aligned}
$$

where $S$ is a matrix convergent to zero.

Since the hypothesis of Theorem 4 is satisfied we get that the periodic boundary value problem (3.4) and (3.6) has a unique solution on $I$.

(ii) We define $T: X \times X \rightarrow X$, for $t \in I$, by

$$
\begin{aligned}
T(u, v)(t) & =\int_{0}^{T} G_{1}(t, s)\left[f_{1}(s, u)+g_{1}(s, v)+\lambda_{1} u-\lambda_{2} v\right] \\
& +G_{2}(t, s)\left[f_{1}(s, v)+g_{1}(s, u)+\lambda_{1} v-\lambda_{2} u\right] d s
\end{aligned}
$$

Then, after some calculation, we get:

$$
\begin{aligned}
& |A(u, v)(t)-T(u, v)(t)| \\
& =\mid \int_{0}^{T} G_{1}(t, s)\left[f(s, u)+g(s, v)+\lambda_{1} u-\lambda_{2} v\right] \\
& \quad+G_{2}(t, s)\left[f(s, v)+g(s, u)+\lambda_{1} v-\lambda_{2} u\right] d s \\
& -\int_{0}^{T} G_{1}(t, s)\left[f_{1}(s, u)+g_{1}(s, v)+\lambda_{1} u-\lambda_{2} v\right] \\
& \quad+G_{2}(t, s)\left[f_{1}(s, v)+g_{1}(s, u)+\lambda_{1} v-\lambda_{2} u\right] d s \mid \\
& \leq \int_{0}^{T} \mid G_{1}(t, s) \\
& \quad \quad\left[\left[f(s, u)+g(s, v)+\lambda_{1} u-\lambda_{2} v\right]-\left[f_{1}(s, u)+g_{1}(s, v)+\lambda_{1} u-\lambda_{2} v\right]\right] \\
& \quad+G_{2}(t, s)\left[\left[f(s, v)+g(s, u)+\lambda_{1} v-\lambda_{2} u\right]\right. \\
& \quad-\left[f_{1}(s, v)+g_{1}(s, u)+\lambda_{1} v-\lambda_{2} u\right] \mid d s \\
& \leq\left(\eta_{1}+\eta_{2}\right)\left(\int_{0}^{T}\left|G_{1}(t, s)\right| d s+\int_{0}^{T}\left|G_{2}(t, s)\right| d s\right) .
\end{aligned}
$$

Taking the supremum after $t \in I$ we obtain that

In a similar way, we get that

$$
d(A(u, v), T(u, v)) \leq\left(\eta_{1}+\eta_{2}\right) \frac{1}{\lambda_{2}-\lambda_{1}} .
$$

$$
d(A(v, u), T(v, u)) \leq\left(\eta_{1}+\eta_{2}\right) \frac{1}{\lambda_{2}-\lambda_{1}} .
$$

Now the conclusion follows by Theorem 4 (iv). 
(iii) By the first part of our proof and by Theorem 4 (vi) we get the conclusion.

Remark 1. From the proof of Theorem 5 and Theorem 4 (ii), (iii) we also get the following conclusions:

(a) the sequence $\left(A^{n}(u, v), A^{n}(v, u)\right)_{n \in \mathbb{N}}$ converges in $X \times X$ to $\left(u^{*}, v^{*}\right)$ as $n \rightarrow$ $\infty$, where

for all $n \in \mathbb{N}^{*}$.

$$
\left(A^{n+1}(u, v), A^{n+1}(v, u)\right):=A^{n}(A(u, v), A(v, u))
$$

(b) we have the following estimation:

$$
\left(\begin{array}{c}
d\left(A^{n}\left(u_{0}, v_{0}\right), u^{*}\right) \\
d\left(A^{n}\left(v_{0}, u_{0}\right), v^{*}\right)
\end{array}\right) \leq S^{n}(I-S)^{-1}\left(\begin{array}{c}
d\left(u_{0}, A\left(u_{0}, v_{0}\right)\right) \\
d\left(v_{0}, A\left(v_{0}, u_{0}\right)\right)
\end{array}\right)
$$

\section{REFERENCES}

[1] G. Allaire and S. M. Kaber, Numerical linear algebra, ser. Texts in Applied Mathematics. New York: Springer, 2008, vol. 55.

[2] T. G. Bhaskar and V. Lakshmikantham, "Fixed point theorems in partially ordered metric spaces and applications," Nonlinear Anal., Theory Methods Appl., vol. 65, no. 7, pp. A, 1379-1393, 2006.

[3] A. Bucur, L. Guran, and A. Petrus sel, "Fixed points for multivalued operators on a set endowed with vector-valued metrics and applications," Fixed Point Theory, vol. 10, no. 1, pp. 19-34, 2009.

[4] A.-D. Filip and A. Petruşel, "Fixed point theorems on spaces endowed with vector-valued metrics," Fixed Point Theory Appl., vol. 2010, p. 15, 2010.

[5] D. Guo and V. Lakshmikantham, "Coupled fixed points of nonlinear operators with applications," Nonlinear Anal., Theory Methods Appl., vol. 11, pp. 623-632, 1987.

[6] V. Lakshmikantham and L. Ćirić, "Coupled fixed point theorems for nonlinear contractions in partially ordered metric spaces," Nonlinear Anal., Theory Methods Appl., vol. 70, no. 12, pp. A, 4341-4349, 2009.

[7] A. I. Perov, "On the Cauchy problem for a system of ordinary differential equations," Priblizhen. Metody Reshen. Differ. Uravn., vol. 2, pp. 115-134, 1964.

[8] A. Petruşel, "Multivalued weakly Picard operators and applications," Sci. Math. Jpn., vol. 59, no. 1, pp. 169-202, 2004.

[9] R. Precup, "The role of matrices that are convergent to zero in the study of semilinear operator systems," Math. Comput. Modelling, vol. 49, no. 3-4, pp. 703-708, 2009.

[10] I. A. Rus, Principles and Applications of the Fixed Point Theory. Dacia: Cluj-Napoca, 1979.

[11] I. A. Rus, "Remarks on Ulam stability of the operatorial equations," Fixed Point Theory, vol. 10, no. 2, pp. 305-320, 2009.

[12] C. Urs, "Hyers-Ulam stability for coupled fixed points of contractive type operators," J. Nonlinear Sci. Appl., to appear.

[13] R. S. Varga, Matrix iterative analysis. 2nd revised and expanded ed, ser. Springer Series in Computational Mathematics. Berlin: Springer, 2000, vol. 27.

\section{Author's address}

\section{Cristina Urs}

Department of Mathematics, Babeş-Bolyai University Cluj-Napoca,, Kogălniceanu Street No.1, 400084, Cluj-Napoca, Romania.

E-mail address: cristina.urs@math.ubbcluj.ro 